\title{
Bertelsmann-Studie deckt Versorgungsdefizite auf
}

— Fast $90 \%$ aller Sterbenden brauchen eine palliative Begleitung - nur $30 \%$ haben sie 2014 erhalten. Trotz der Einführung des Rechts der "Spezialisierten Ambulanten Palliativversorgung" (SAPV) auf eigenen Wunsch zu Hause zu sterben, zeigt sich, dass die Versorgungswirklichkeit hierzulande weit davon entfernt ist. Das macht eine Studie der Bertelsmann-Stiftung zum Thema Ausbau der Palliativversorgung in Deutschland deutlich. Untersucht wurden dabei die Versorgungsleistungen für Menschen während der letzten Lebensphase.

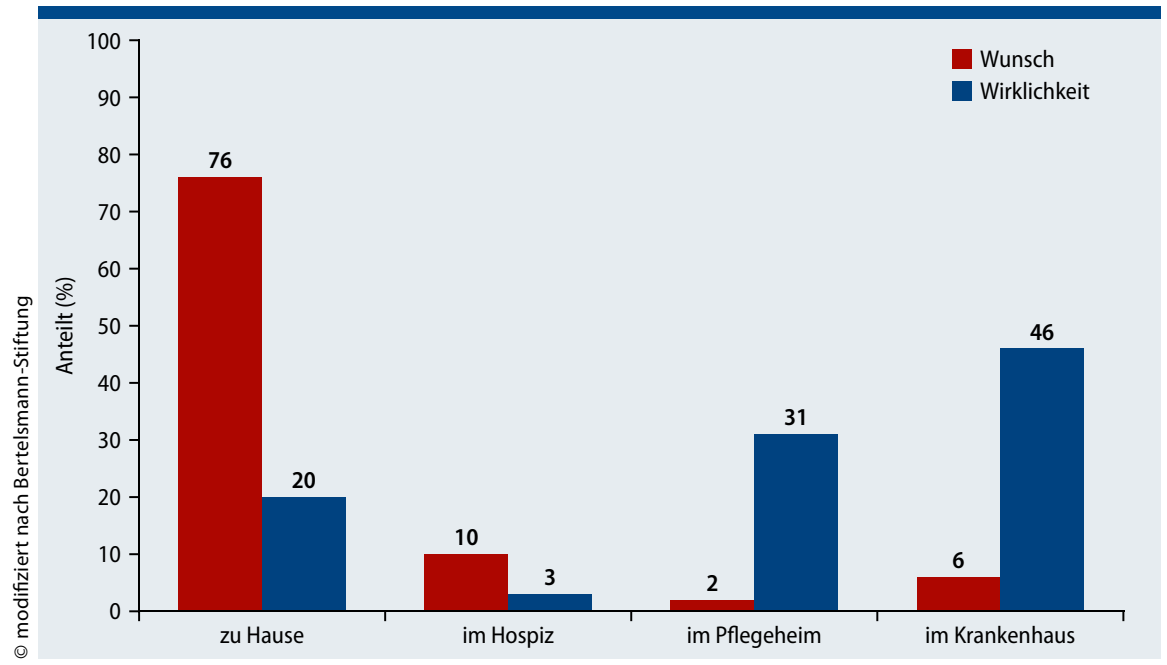

\section{Mehr Versorgungstransparenz}

\section{Klassifizierung von schmerzmedizinischen Einrichtungen}

— Die Fachgesellschaften und Verbände in der Schmerzmedizin haben sich auf einen Katalog geeinigt, in dem erstmals Strukturund Qualitätskriterien für unterschiedliche schmerzmedizinische Einrichtungen formuliert sind. Die Versorgungslandschaft ergebe derzeit ein völlig uneinheitliches Bild, erläuterte der Präsident der DGS Dr. Gerhard Müller-Schwefe bei einer von Mundipharma unterstützten Fachveranstaltung in Stuttgart.

Jetzt soll mehr Transparenz entstehen. Die "Gemeinsame Kommission der Fachgesell-
Die Studie zeigt, dass jeder zweite ältere Deutsche im Krankenhaus stirbt, obwohl $75 \%$ der Menschen den Tod daheim vorziehen würden. Die Stiftung fordert, dass die ambulante Palliativversorgung für ein würdevolles Sterben zu Hause weiter ausgebaut werden muss.

„Die Planung neuer Versorgungsangebote sollte sich an dem Wunsch der allermeisten Menschen ausrichten, ihre letzten Lebenstage zu Hause zu verbringen", sagte Dr. Brigitte Mohn, Vorstand der Bertelsmann Stiftung.

Christoph Fuhr

Muskelschmerzen unter Statinen

\section{Dosisreduktion oder Präparatewechsel?}

— Bei der Primär- und Sekundärprävention vaskulärer Ereignisse sind Statine unverzichtbar. So werden in Deutschland zur Zeit fünf Millionen Patienten mit einer solchen Substanz behandelt. Der häufigste Grund für das Absetzen eines Statins seien Muskelschmerzen und dies sei prognostisch relevant, sagte Professor Ulrich Laufs von der kardiologischen Universitätsklinik in Homburg/Saar auf dem DGN-Kongress 2015.

Doch nicht jeder Muskelschmerz ist medikamentös induziert. Von einer Statinmyopathie ist dann auszugehen, wenn die Symptome innerhalb von vier Wochen nach Beginn der Statineinnahme auftreten und sich innerhalb von vier Wochen nach Absetzen des Medikaments wieder zurückbilden. Doch wie sollte man vorgehen, wenn ein Patient nach Einleitung einer Statintherapie über Muskelschmerzen klagt und andere Ursachen ausgeschlossen sind? „Zunächst sollte man die Therapie für zwei bis vier Wochen unterbrechen", so die Empfehlung von Laufs. Anschließend sollte man die Therapie wieder aufnehmen. Bei einem Teil der Patienten treten die Muskelschmerzen dann nicht mehr auf und man kann stufenweise auftitrieren, bis der Zielwert erreicht ist. Führt diese Reexposition aber erneut zu Beschwerden, so sollte ein anderes potentes Statin in einer niedrigen Dosierung gewählt werden. Die Dosis kann dann langsam erhöht werden, bis erneut Beschwerden auftreten. So lässt sich die gerade noch verträgliche Dosis ermitteln. Von einer Statinintoleranz sollte man erst dann sprechen, wenn die kleinste Dosis von zwei unterschiedlichen Statinen nicht toleriert wird.

Dr. Peter Stiefelhagen

schaften und Verbände in der Schmerzmedizin" hat schmerzmedizinische Einrichtungen definiert und festgelegt, welche Kriterien sie erfüllen müssen: So gibt es etwa Einrichtungen, von denen erwartet wird, dass der Leiter jährlich 40 Stunden schmerzmedizinische Fortbildung und zehn offene Schmerzkonferenzen nachweisen muss. Wichtig, so Müller-Schwefe, sei es, dass die Klassifizierung Strukturen definiere, die im bestehenden Versorgungssystem umsetzbar und überprüfbar sind.

Christoph Fuhr

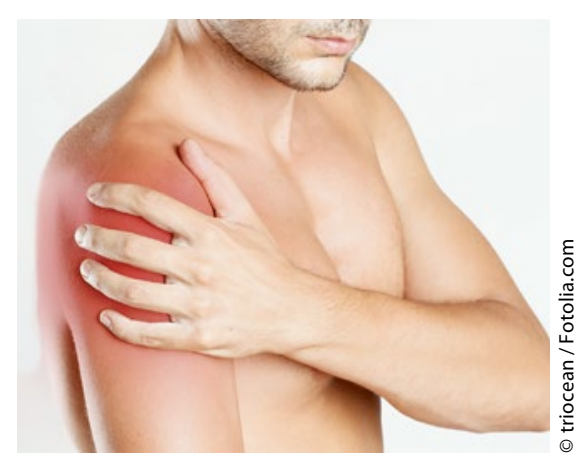

